\title{
Comparison among four calibrated meta-heuristic algorithms for solving a type-2 fuzzy cell formation problem considering economic and environmental criteria
}

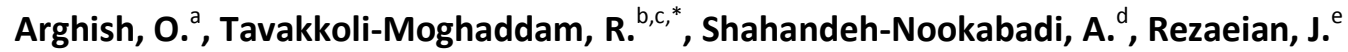 \\ ${ }^{a}$ Department of Industrial Engineering, Science and Research Branch, Islamic Azad University, Tehran, Iran \\ ${ }^{\mathrm{b}}$ School of Industrial Engineering, College of Engineering, University of Tehran, Tehran, Iran \\ 'LCFC, Arts et Métiers Paris Tech, Metz, France \\ dDepartment of Industrial and Systems Engineering, Isfahan University of Technology, Isfahan, Iran \\ e Department of Industrial Engineering, Mazandaran University of Science and Technology, Babol, Iran
}

\begin{abstract}
A B S T R A C T
In this paper, a mathematical model is proposed using economic and environmental criteria for a type-2 fuzzy (T2F) cell formation (CF) problem emphasizing the effect of the man-machine relationship aspect. This model aims to show the use of this aspect in CF to minimize the costs of processing, material movement, energy loss, and tooling. For this purpose, a two-stage defuzzification procedure is used to convert the T2F variable into a crisp value. Due to NP-hardness of the model and problem, a genetic algorithm (GA) is used to derive the appropriate solutions. Furthermore, because there is no any existing benchmark to validate the performance of the proposed model, three tuned meta-heuristic algorithms, namely, differential evolution (DE), harmony search (HS) and particle swarm optimization (PSO), are proposed and used. The present research uses the Taguchi method to adjust the parameters in the four proposed algorithms. Furthermore, 15 examples are used to validate the presented model. The results show that PSO is the most appropriate algorithm for solving the model.
\end{abstract}

\author{
ARTICLE INFO \\ Keywords: \\ Cell formation; \\ Environmental factor; \\ Genetic algorithm; \\ Particle swarm optimization; \\ Harmony search; \\ Differential evolution \\ *Corresponding author: \\ tavakoli@ut.ac.ir \\ (Tavakkoli-Moghaddam, R.) \\ Article history: \\ Received 29 June 2017 \\ Revised 22 January 2018 \\ Accepted 20 February 2018
}

\section{References}

[1] Rafiei, H., Ghodsi, R. (2013). A bi-objective mathematical model toward dynamic cell formation considering labor utilization, Applied Mathematical Modelling, Vol. 37, No. 4, 2308-2316, doi: 10.1016/j.apm.2012.05.015.

[2] Greene, T.J., Sadowski, R.P. (1984). A review of cellular manufacturing assumptions, advantages and design techniques, Journal of Operations Management, Vol. 4, No. 2, 85-97, doi: 10.1016/0272-6963(84)90025-1.

[3] Mehdizadeh, E., Rahimi, V. (2016). An integrated mathematical model for solving dynamic cell formation problem considering operator assignment and inter/intra cell layouts, Applied Soft Computing, Vol. 42, 325-341, doi: 10.1016/j.asoc.2016.01.012.

[4] Majazi Dalfard, V. (2013). New mathematical model for problem of dynamic cell formation based on number and average length of intra and intercellular movements, Applied Mathematical Modelling, Vol. 37, No. 4, 1884-1896, doi: 10.1016/j.apm.2012.04.034.

[5] Deljoo, V., Mirzapour Al-e-hashem, S.M.J., Deljoo, F., Aryanezhad, M.B. (2010). Using genetic algorithm to solve dynamic cell formation problem, Applied Mathematical Modelling, Vol. 34, No. 4, 1078-1092, doi: 10.1016/j.apm. 2009.07.019.

[6] Bagheri, M., Bashiri, M. (2014). A new mathematical model towards the integration of cell formation with operator assignment and inter-cell layout problems in a dynamic environment, Applied Mathematical Modelling, Vol. 38, No. 4, 1237-1254, doi:10.1016/j.apm.2013.08.026. 
[7] Xu, H., Bao, Z.R., Zhang, T. (2017). Solving dual flexible job-shop scheduling problem using a Bat Algorithm, Advances in Production Engineering \& Management, Vol. 12, No. 1, 5-16, doi: 10.14743/apem2017.1.235.

[8] Zupan, H., Herakovic, N., Zerovnik, J., Berlec, T. (2017). Layout optimization of a production cell, International Journal of Simulation Modelling, Vol. 16, No. 4, 603-616, doi: 10.2507/IJSIMM16(4)4.396.

[9] Nie, X.D., Chen, X.D., Chen, X. (2016). Simulation study of flexible manufacturing cell based on token-oriented Petri net model, International Journal of Simulation Modelling, Vol. 15, No. 3, 566-576, doi: 10.2507/IJSIMM15(3) C014.

[10] Mahdavi, I., Mahdavi-Amiri, N., Naderi, F., Paydar, M.M. (2017). Cell formation configuration using interval type-2 fuzzy interactional interests among workers, International Journal of Operational Research, Vol. 30, No. 2, doi: 10.1504/IJOR.2017.10007273.

[11] Stocchetti, A. (2012). The sustainable firm: From principles to practice, International Journal of Business and Management, Vol. 7, No. 21, 34-47, doi: /10.5539/ijbm.v7n21p34.

[12] Lozano, R. (2008). Envisioning sustainability three-dimensionally, Journal of Cleaner Production, Vol. 16, No. 17, 1838-1846, doi: 10.1016/i.jclepro.2008.02.008.

[13] Niakan, F., Baboli, A., Moyaux, T., Botta-Genoulaz, V. (2016). A bi-objective model in sustainable dynamic cell formation problem with skill-based worker assignment, Journal of Manufacturing Systems, Vol. 38, 46-62, doi: 10.1016/j.jmsy.2015.11.001.

[14] Niakan, F., Baboli, A., Moyaux, T., Botta-Genoulaz, V.A. (2015). A new multi-objective mathematical model for dynamic cell formation under demand and cost uncertainty considering social criteria, Applied Mathematical Modelling, Vol. 40, No. 4, 2674-2691, doi: 10.1016/i.apm.2015.09.047.

[15] Niakan, F., Baboli, A., Moyaux, T., Botta-Genoulaz, V. (2014). A new bi-objective mathematical model for sustainable dynamic cellular manufacturing systems, In: Proceedings of the IEEE International Conference on Industrial Engineering and Engineering Management, Selangor, Malaysia, 938-942, doi: 10.1109/IEEM.2014.7058776.

[16] Niakan, F., Baboli, A., Moyaux, T., Botta-Genoulaz, V. (2016). A bi-objective model in sustainable dynamic cell formation problem with skill-based worker assignment, Journal of Manufacturing Systems, Vol. 38, 46-62, doi: 10.1016/i.jmsy.2015.11.001.

[17] Safaei, N., Saidi-Mehrabad, M., Tavakkoli-Moghaddam, R., Sassani, F. (2008). A fuzzy programming approach for a cell formation problem with dynamic and uncertain conditions, Fuzzy Sets and Systems, Vol. 159, No. 2, 215236, doi: 10.1016/i.fss.2007.06.014.

[18] Qin, R., Liu, Y.K., Liu, Z.Q. (2011). Methods of critical value reduction for type-2 fuzzy variables and their applications, Journal of Computational and Applied Mathematics, Vol. 235, No. 5, 1454-1481, doi: 10.1016/i.cam.2010. $\underline{08.031}$.

[19] Miller, S., John, R. (2010). An interval type-2 fuzzy multiple echelon supply chain model, Knowledge-Based Systems, Vol. 23, No. 4, 363-368, doi: 10.1016/j.knosys.2009.11.016.

[20] Kundu, P., Kar, S., Maiti, M. (2014). Fixed charge transportation problem with type-2 fuzzy variables, Information Sciences, Vol. 255, 170-186, doi: 10.1016/i.ins.2013.08.005.

[21] Niebel, B.W. (1982). Motion and time study, Homewood, Irwin, USA.

[22] de Castro Hilsdorf, W., de Mattos, C.A., de Campos Maciel, L.O. (2017). Principles of sustainability and practices in the heavy-duty vehicle industry: A study of multiple cases, Journal of Cleaner Production, Vol. 141, 1231-1239, doi: 10.1016/j.jclepro.2016.09.186.

[23] Wang, L.X. (1996). A course in fuzzy systems and control, Prentice-Hall International, Inc., U.S.A.

[24] Holland, J.H. (1975). Adaptation in natural and artificial systems: An introductory analysis with applications to biology, control and artificial intelligence, MIT Press Cambridge, MA, USA.

[25] Mousavi, S.M., Hajipour, V., Niaki, S.T.A., Aalikar, N. (2014). A multi-product multi-period inventory control problem under inflation and discount: A parameter-tuned particle swarm optimization algorithm, The International Journal of Advanced Manufacturing Technology, Vol. 70, No. 9-12, 1739-1756, doi: 10.1007/s00170-013-5378-y.

[26] Noktehdan, A., Karimi, B., Husseinzadeh Kashan, A. (2010). A differential evolution algorithm for the manufacturing cell formation problem using group-based operators, Expert Systems with Applications, Vol. 37, No. 7, 4822-4829, doi: 10.1016/j.eswa.2009.12.033.

[27] Storn, R., Price, K. (1997). Differential evolution - A simple and efficient heuristic for global optimization over continuous spaces, Journal of Global Optimization, Vol. 11, No. 4, 341-359, doi: 10.1023/A:1008202821328.

[28] Sahin, O., Akay, B. (2016). Comparisons of metaheuristic algorithms and fitness functions on software test data generation, Applied Soft Computing, Vol. 49, 1202-1214, doi: 10.1016/j.asoc.2016.09.045.

[29] Kennedy, J., Eberhart, R. (1995). Particle swarm optimization, In: Proceedings of the IEEE International Conference on Neural Networks, Perth, WA, Australia, 1942-1948.

[30] Pant, M., Thangaraj, R., Abraham, A. (2009). Particle swarm optimization: Performance tuning and empirical analysis, Foundations of Computational Intelligence, Vol. 3, 101-128, doi: 10.1007/978-3-642-01085-9 5.

[31] Geem, Z.W., Kim, J.H., Loganathan, G.V. (2001). A new heuristic optimization algorithm: Harmony search, Simulation, Vol. 76, No. 2, 60-68, doi: 10.1177/003754970107600201.

[32] Askarzadeh, A., Zebarjadi, M. (2014). Wind power modeling using harmony search with a novel parameter setting approach, Journal of Wind Engineering and Industrial Aerodynamics, Vol. 135, 70-75, doi: 10.1016/i.jweia. 2014.10.012. 